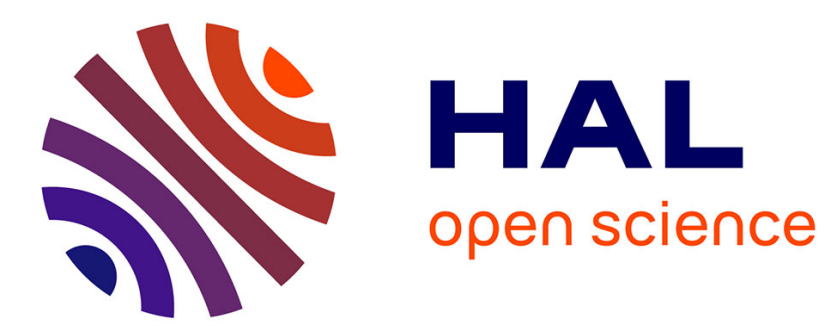

\title{
Alternate extraction and acceleration of positive and negative ions from a gridded plasma source
}

\author{
Trevor Lafleur, D.V. Rafalskyi, Ane Aanesland
}

\section{To cite this version:}

Trevor Lafleur, D.V. Rafalskyi, Ane Aanesland. Alternate extraction and acceleration of positive and negative ions from a gridded plasma source. Plasma Sources Science and Technology, 2015, 24 (1), pp.15005. 10.1088/0963-0252/24/1/015005 . hal-01104903

\section{HAL Id: hal-01104903 \\ https://hal.sorbonne-universite.fr/hal-01104903}

Submitted on 19 Jan 2015

HAL is a multi-disciplinary open access archive for the deposit and dissemination of scientific research documents, whether they are published or not. The documents may come from teaching and research institutions in France or abroad, or from public or private research centers.
L'archive ouverte pluridisciplinaire HAL, est destinée au dépôt et à la diffusion de documents scientifiques de niveau recherche, publiés ou non, émanant des établissements d'enseignement et de recherche français ou étrangers, des laboratoires publics ou privés. 


\title{
Alternate extraction and acceleration of positive and negative ions from a gridded plasma source
}

\author{
T. Lafleur ${ }^{1,2}$, D. Rafalskyi ${ }^{1}$, and A. Aanesland ${ }^{1}$ \\ ${ }^{1}$ Laboratoire de Physique des Plasmas, CNRS, Sorbonne Universités, UPMC Univ \\ Paris 06, Univ Paris-Sud, Ecole Polytechnique, 91128 Palaiseau, France \\ 2 ONERA-The French Aerospace Lab, 91120 Palaiseau, France \\ E-mail: trevor.lafleur@lpp.polytechnique.fr
}

\begin{abstract}
By applying a square-wave voltage with frequencies between $10 \mathrm{kHz}$ to $1 \mathrm{MHz}$ to a set of grids terminating an ion-ion plasma source, we experimentally demonstrate the alternate extraction and acceleration of high energy (100's of eV) positive and negative ion beams. In addition, the ratio of positive-to-negative ion beam current can be controlled by adjusting the applied square-wave duty cycle. Temporallyresolved floating potential measurements of a target show that the downstream potential can be controlled and sufficiently reduced at high applied frequencies $(\sim$ $200 \mathrm{kHz}$ ), indicating that space-charge compensation can be achieved to prevent beam stalling.
\end{abstract}




\section{Introduction}

For the fabrication of a number of microelectronic components (such as gigabit random access memories [1]) there is a need to produce highly anisotropic silicon etch patterns with high-aspect ratio features $[1,2,3]$. In many inductively or capacitively coupled plasma sources (ICPs or CCPs), ions strike the material substrate with well-directed energies, while the plasma electrons have a more isotropic distribution. As a result, these electrons can produce side wall charging in etch features $[2,3]$, which can cause deflection of ion trajectories and hence undesirable side-wall etching; a process leading to notching or re-entrant profiles (see for example Ref. [3]). The use of almost electron-free ion-ion plasmas has been proposed as a possible alternative etch method to avoid this problem $[1,4,5]$. Here both positive and negative ions are successively accelerated $[6,7,8,9]$ towards a substrate surface (by applying a time-varying bias voltage), thus allowing both species to participate in the etch process, and more importantly, reducing charging damage on the side walls. The formation of ion-ion plasmas has been demonstrated in the after-glow of pulsed plasmas [6, 10], electron-beam produced plasmas [11], and in ICP sources containing a magnetic filter [12].

The extraction of positive and negative ions from ion-ion plasmas has also been proposed as an electric propulsion concept known as PEGASES [13] (Plasma propulsion with Electronegative GASES). Here a plasma source produces an ion-ion plasma by using a magnetic barrier to impede downstream electron transport; a process which reduces the electron thermal conductivity (and hence temperature $[14,15]$ ) and favours negative ion formation by electron-neutral attachment. By then applying a time-varying squarewave bias to a set of grids [16], successive high-energy beams of positive and negative ions are extracted without the need for a separate electron-emitting neutralizer. Such neutralizers are needed in most current electric propulsion systems to ensure charge neutrality of the spacecraft, and to prevent beam stalling (due to the space-charge of the ion beam itself) $[17,18]$. This prevention of stalling is usually referred to as beam neutralization $[17,18]$, since the uncompensated space-charge is "neutralized" by the injection of electrons to produce a quasi-neutral plasma exhaust. The concept of using negative ions instead of electrons for beam neutralization was also independently proposed more than 50 years ago $[17,19]$, although at that time an efficient negative ion source proved difficult to develop, and so further work essentially stopped. Here a thruster with a mosaic-like structure of tightly-packed inter-mixed positive and negative ion guns was envisioned, with the natural spreading of each beam utilized to ensure rapid mixing of the positive and negative ions, and hence compensation of any unbalanced space-charge (while producing thrust with both ionic species). This concept was initially developed to address concerns related to the use of electrons for beam neutralization [17]. More generally though, electron-free thruster exhaust plumes could prove important for future mission applications, such as for space debris removal schemes [20], where electron interactions with ambient magnetic fields could increase beam divergence and reduce the efficiency of such schemes. 
Experimental validation of the more recent PEGASES concept has so far been strongly limited to DC extraction (or very low frequencies of $<20 \mathrm{kHz}$, which give an essentially DC plasma response) where the downstream space charge of the accelerated beams has not been compensated by the extracted particles themselves, and spacecraft charge neutrality has not been addressed (i.e. nominally it is not known if equal positive and negative ion currents can be extracted). In this paper we explicitly address these issues and experimentally demonstrate that by applying a sufficiently high-frequency bias voltage to an ion-ion plasma source, positive and negative ions can be successively extracted and accelerated while maintaining good downstream beam neutralization.

\section{Description of experiment}

The PEGASES plasma source (see Fig. 1) used here consists of a $12 \mathrm{~cm}$ long, rectangular aluminium tube with dimensions of $8 \mathrm{~cm}$ by $12 \mathrm{~cm}$. The tube is closed at one end by a $2 \mathrm{~mm}$ thick ceramic window and a 7-turn ferrite-embedded (Ferroxcube 4C65) rf antenna operated at $4 \mathrm{MHz}$, and terminated at the opposite end by a set of two grids attached to a PEEK (PolyEther Ether Ketone) support structure. The identical stainless steel grids are $2 \mathrm{~mm}$ apart with a thickness of $1 \mathrm{~mm}$, an aperture diameter of $2.5 \mathrm{~mm}$, and a transparency of about $60 \%$. The grids were manufactured simultaneously, and so the apertures are aligned. Placed inside the aluminium tube is an additional Pyrex tube (see Fig. 1), while $7.5 \mathrm{~cm}$ away from the ceramic window are a set of neodymium permanent magnets (with a maximum field strength of about $245 \mathrm{G}$ ). The plasma source is attached to a larger $70 \mathrm{~cm}$ long vacuum chamber with a diameter of $60 \mathrm{~cm}$, and is pumped with a rotary and turbo-molecular pump combination. The pressure in this larger chamber is measured with baratron and ion gauges.

Neutral gas is injected on one side of the plasma source through a series of 8 holes, and consists of a 50-50 mixture of argon $(13 \mathrm{SCCM}=0.39 \mathrm{mg} / \mathrm{s})$ and $\mathrm{SF}_{6}$ $(13 \mathrm{SCCM}=1.34 \mathrm{mg} / \mathrm{s})$. At these flow rates the downstream vacuum chamber pressure is 0.3 mTorr, while the pressure inside the plasma source is around $1 \mathrm{mTorr}$. Rf power is fed to the symmetrically driven antenna via a matching network composed of a transmission-line transformer and a series of variable capacitors. For all experiments performed here, the total applied power is $220 \mathrm{~W}$, which accounting for power losses in the antenna (determined from measurements of the antenna current and the total applied rf power), corresponds to a discharge power of about $100 \mathrm{~W}$. Ion extraction and acceleration is achieved using a square-wave voltage applied to the first (or screen) grid with a PG2-500 single-phase invertor (constructed at the KhNU, Ukraine). The second (or accel) grid is grounded. During positive or negative ion extraction, previous results [16] have indicated that the physical collection area of the screen grid may be insufficient to maintain current balance within the source. This problem is solved by connecting an additional metal electrode near the screen grid (and biased at the grid potential) as indicated in Fig. 1.

Measurement of the extracted positive and negative ions is performed with a 
specially designed magnetized retarding field energy analyzer (MRFEA) previously described [16]. In brief, the single-grid probe suppresses electron collection via a highstrength ( $\sim 400 \mathrm{G})$ localised magnetic field at the entrance of the probe. The probe is mechanically attached to the accel grid, and located near one of the corners. To estimate the degree of beam space-charge compensation, a large movable floating metal target (with a diameter of $15 \mathrm{~cm}$ ) is placed $5-10 \mathrm{~cm}$ downstream of the accel grid, and the floating potential measured with a high-voltage probe connected to an oscilloscope.

\section{Results}

\subsection{Alternate extraction and acceleration}

Under the present operating conditions, a strong ion-ion plasma (with an electronegativity of a few 1000's) is formed in the region between the magnetic filter and the screen grid [15]. The filter significantly reduces the electron temperature (below about $1 \mathrm{eV}$ ), and facilitates negative ion formation due to attachment. Close to the screen grid the negative and positive ions have essentially identical densities $\left(\sim 1 \times 10^{17} \mathrm{~m}^{-3}\right)$ and temperatures $(\sim 0.1 \mathrm{eV})[15]$, which gives current densities (measured on the inner surface of the screen grid with a planar probe [16]) of about $1 \mathrm{~mA} / \mathrm{cm}^{2}$. Since the only potential reference that the plasma is in direct contact with is the screen grid (together with the additional electrode at the same potential), the plasma potential follows the applied grid bias when electron leakage-current effects are eliminated [16]. Figure 2 shows the measured ion beam energy and current density as a function of the applied square-wave frequency for a voltage amplitude of $350 \mathrm{~V}$. Also shown is a representative ion-flux distribution function (IF-DF; inset figure in Fig. 2 (a)) obtained from the derivative (with respect to the voltage) of the measured current-voltage (IV) curve from the MRFEA. This time-averaged distribution shows strong positive and negative ion peaks centered at approximately the applied voltage amplitude. A low-energy peak centered at $0 \mathrm{eV}$ is also present due to secondary effects in the analyzer (such as secondary electron emission, or the surface production of negative ions). The beam energy is almost constant with applied frequency in the range tested, but the negative ion energy is found to be very slightly lower $(5-10 \%)$ than the nominal applied value.

Determination of the exact positive and negative ion composition in the present system is difficult, particularly since $\mathrm{SF}_{6}$ plasmas typically display a large range of species. However, fluid simulations of the present system in pure $\mathrm{SF}_{6}$ [21] show that the dominant positive ion species is $\mathrm{SF}_{5}{ }^{+}$, while the dominant negative ion species are $\mathrm{SF}_{6}{ }^{-}$and $\mathrm{F}^{-}$. Previous Langmuir probe measurements [15] (made on-axis) have observed almost symmetric negative and positive ion saturation regions in the measured IV characteristics, and a good fit to the data using a developed model was obtained for equal positive and negative ion masses of $127 \mathrm{AMU}$ (which is identical to that for $\mathrm{SF}_{5}{ }^{+}$, and similar to that for $\mathrm{SF}_{6}{ }^{-}$). This therefore suggests that the dominant ion species are 
$\mathrm{SF}_{5}{ }^{+}$and $\mathrm{SF}_{6}{ }^{-}$; although given that a gas mixture of $\mathrm{Ar}$ and $\mathrm{SF}_{6}$ is being used here, some fraction of $\mathrm{Ar}^{+}$ions is also expected.

The effect on beam propagation due to ion-neutral collisions with the background neutral gas (located both between the extraction grids and in the downstream vacuum chamber) can be estimated by calculating the ion mean free path. Ion-neutral collision cross-sections are typically not well known for $\mathrm{SF}_{6}$, but taking representative values (at an energy of $350 \mathrm{eV}$ ) for $\mathrm{SF}_{6}{ }^{-}{ }^{-} \mathrm{SF}_{6}$ collisions from Ref. [22], we obtain a cross-section of about $\sigma_{i} \sim 3 \times 10^{-19} \mathrm{~m}^{2}$. As mentioned in Section 2, during operation the pressure in the plasma source is about $1 \mathrm{mTorr}$, while the downstream vacuum chamber pressure is $0.3 \mathrm{~m}$ Torr. Using the worst case pressure of 1 mTorr, the mean free path of an ion is estimated to be around $10 \mathrm{~cm}$. Given that the neutral pressure rapidly decays outside of the plasma source (hence resulting in a larger mean free path), the ion beams can propagate relatively far from the source before being scattered and thermalized by ionneutral collisions. Positive and negative ion beams can still be observed at a distance of about $10 \mathrm{~cm}$ from the source, as measured with a second MRFEA embedded in the downstream target (which is grounded for this test). An example of an IF-DF is shown in Fig. 3 at a frequency of $250 \mathrm{kHz}$ and an applied voltage amplitude of $350 \mathrm{~V}$.

Figure 2 (b) shows the time-averaged beam current densities obtained with the MRFEA attached to the accel grid. Here the beam current density is defined as that resulting from a 1- $\sigma$ width from the approximately Gaussian-shaped beams. As the frequency increases, both positive and negative ion beam current densities decrease slightly until a minimum at about $850 \mathrm{kHz}$. This decrease of current density with increasing frequency can be expected because of the larger number of rf cycles (and hence the larger number of waveform polarity changes and therefore ion extraction transients) within a given time interval. For the above measurements, the square-wave duty cycle is fixed at 50\%, which gives a negative ion beam current density about half that of the positive ion beam current density.

For the applied voltage amplitude $\left(V_{0}\right)$, gap length $(L$; and thickness of both grids, $2 t)$, and approximate ion mass $\left(M_{i} \approx 127 \mathrm{AMU}\right)$, the ion transit time through the grids $(\tau$; assuming that the ions start from rest) can be estimated from

$$
\tau=\sqrt{\frac{2 M_{i}(L+2 t)^{2}}{q V_{0}}}
$$

where $q$ is the magnitude of the ion charge (we assume here singly charged ions). This gives a value of $\tau=0.35 \mu \mathrm{s}$. If we set this transit time equal to half an applied square waveform period, then we obtain a frequency of about $1.4 \mathrm{MHz}$, which is similar to the frequency at the minimum in Fig. 2 (b). By comparison, the ion plasma frequency, $f_{p i}$, is about $6 \mathrm{MHz}$, as calculated from

$$
f_{p i}=\frac{\omega_{p i}}{2 \pi}=\frac{1}{2 \pi} \sqrt{\frac{q^{2} n_{i}}{\epsilon_{0} M_{i}}}
$$


where $\omega_{f i}$ is the angular frequency, $n_{i}$ is the ion density near the grids $\left(n_{i} \sim 1 \times 10^{17} \mathrm{~m}^{-3}\right)$, and $\epsilon_{0}$ is the permittivity of free space. Hence the extraction frequencies used here are much lower than the ion plasma frequency.

\subsection{Duty cycle}

Figure 4 shows the ion beam current densities as a function of the square-wave duty cycle. Here, as also indicated in Fig. 2 (b), the positive and negative ion currents are not equal at a duty cycle of $50 \%$. This can occur if: (1) the extracted currents are not uniform across the grid surface, (2) the positive and negative ions have different masses, or (3) if some fraction of electrons are co-extracted with the negative ions. Although the laboratory plasma source can still maintain current balance, since the non-extracted ion species is drawn to ground via the screen grid (and hence completes the circuit with the extracted ion species to the larger vacuum chamber), for a thruster operating in space, this would present a problem, since equal negative and positive ion fluxes are required. As Fig. 4 shows however, the extracted beam current densities can be controlled by adjusting the square-wave duty cycle. In particular, for this set of operating conditions, equal currents of positive and negative ions can be extracted at a duty cycle of about $35 \%$. In Ref. [23] it was observed that due to insulating film deposition on the screen grid during positive ion extraction, the plasma potential (and hence positive ion beam energy), decreases with time. This occurs because of a voltage drop across the insulating film, and hence a reduction in the effective accelerating voltage. The insulating film can however be cleaned by positive ion bombardment during negative ion extraction; a process that is further improved at lower duty cycles, and which hence fits in well with the lower duty cycle requirement for equal positive and negative ion beam currents found above.

\subsection{Beam neutralization}

In addition to ensuring that a thruster can maintain charge neutrality by emitting equal positive and negative currents, it is also important that the space-charge of any extracted ion beams be "neutralized". In this regard (as already mentioned in Section 1) we use a similar definition of beam neutralization to that used in the electric propulsion community $[17,18]$, where the space-charge of extracted positive ions is "neutralized" by emitting electrons from a hollow cathode. Note that by beam neutralization we do not explicitly mean that the positive and negative ions recombine in the downstream region to form neutral particles. Without compensating the space-charge of the extracted ions, the beams will stall downstream [17], hence producing a significant decrease in thrust and performance. In the present system, which does not make use of an electronemitting neutralizer, an important question arises of whether beam neutralization can be achieved. The original proposal for this concept involves alternatively extracting and accelerating positive and negative ions at a sufficiently high frequency so that any 
uncompensated beam space-charge is either substantially reduced, or that the oppositely charged "beam packets" mix in the downstream region to produce a quasi-neutral exhaust.

A measure of beam neutralization can be made by observing the potential of a floating target which is placed $5 \mathrm{~cm}$ downstream of the accel grid within the beam plume. The level of target charging (both the maximum and time-varying potentials) then serve as an indication of the degree of downstream beam quasi-neutrality. Figure 5 (a) and (b) shows the time-varying floating potential on the target, together with the measured voltage applied to the screen grid. At low frequencies $(\sim 20 \mathrm{kHz})$, during the positive extraction, the target clearly charges up, and the floating potential reaches the beam potential. This indicates that the downstream beam is not quasi-neutral, and that there is a significant fraction of uncompensated space charge. At much higher frequencies however $(\sim 250 \mathrm{kHz})$, there is now insufficient time for the target to charge up, and the maximum potential is significantly lower. This also suggests that the level of uncompensated space charge in the downstream region has been reduced. Figure 5 (c) shows the maximum target potential as a function of applied frequency, where it is seen that for frequencies above about $80 \mathrm{kHz}$, the target potential rapidly decreases with applied frequency. Thus the alternate extracted beams can be well "neutralized" for sufficiently high applied frequencies.

\subsection{Co-extracted electrons}

In the discussion in the previous section, we have ignored the negative portion of the extraction cycle. Observation of this in Fig. 5 shows that while the behaviour at high frequency is not so dissimilar from the positive portion, at low frequencies there is a significant difference. In particular, when the negative polarity portion begins, the target is observed to almost instantaneously discharge. Thereafter, the magnitude of the floating potential does not reach the negative ion beam potential, but remains considerably lower. This behaviour suggests the presence of co-extracted electrons from the plasma source during the negative cycle, as well as the production of a secondary downstream plasma. This is confirmed by visual observations during DC negative ion extraction, where downstream light emission is seen (which is not present during DC positive ion extraction). The extraction of high-energy electrons can result in the production of a secondary plasma in the downstream region due to ionization of the background gas. This can then change the beam neutralization properties, and distort the floating potential measurements of the target, since additional charged particles strike the target.

Particle-in-cell (PIC) simulations [24, 25] in rectangular plasma sources containing a magnetic filter have demonstrated that due to the rectangular geometry, $\mathbf{E} \times \mathbf{B}$ drifts can compromise the ability of the filter to impede electron transport, and consequently, electrons are able to cross the filter on one side of the source. This $\mathbf{E} \times \mathbf{B}$ drift results from the interaction of the sheath/pre-sheath electric fields at the boundaries of the plasma 
source, and the applied filter magnetic field. In particular, for the present system, it is the sheath/pre-sheath electric fields in the z-direction (into/out-of the page in Fig. 1) which are responsible for these drifts, since on one side of the source a drift is directed away from the extraction grids, while on the opposite side a drift is directed towards the extraction grids. The electron current loss profile to the screen grid is then strongly asymmetric [24], and skewed towards the side of the plasma source where the drift is towards the grids.

To investigate the importance of $\mathbf{E} \times \mathbf{B}$ drifts and their role in producing coextracted electrons, current measurements are made inside the plasma source using two planar probes attached to the screen grid. Because of accessibility issues, these measurements are made with a second (but almost identical) plasma source connected to a different vacuum chamber (previously described in Ref. [26]). For these measurements the ion acceleration system is not connected and the extraction grids are replaced with a single grounded stainless grid with a transparency of less than $5 \%$. Because of the significantly reduced pumping conductance of the plasma source due to the presence of this grid, the $\mathrm{Ar}_{-} \mathrm{SF}_{6}$ flow rates are reduced to about $0.5 \mathrm{SCCM}$ each in order to obtain similar neutral gas densities as the original plasma source. The two planar probes used are identical with an area of around $2 \mathrm{~cm}^{2}$, and are placed symmetrically about the vertical axis-of-symmetry (see the schematic in Fig. 6) on the inner surface of the screen grid (but insulated from the grid itself). The probe current is then measured as a function of applied probe bias voltage. Measurements are made both with the original magnetic field configuration, as well as with the field reversed (which would reverse the direction of any $\mathbf{E} \times \mathbf{B}$ drifts).

Results of the measured current on both sides of the source for the two field configurations are shown in Fig. 6. As seen, with the original field, the IV curve from the left-hand side (LHS; when viewing the probes from inside the plasma source looking downstream, as in the schematic in Fig. 6) probe is almost exactly symmetric, with equal positive and negative ion saturation regions; as expected for an ion-ion plasma. However, at the right-hand side (RHS) of the source, the magnitude of the positive portion of the IV curve is significantly larger (by about a factor of about 3) than the negative portion. In addition, the positive ion saturation current is seen to be about 1.5 times higher than the positive ion saturation current measured at the LHS. These observations suggest that there are electrons present on this side of the source, which would both increase the measured current for positive biases, and could also be expected to increase the positive ion current due to a combination of additional ionization, an increase in the effective Bohm velocity from that present in a pure ion-ion plasma [9], or due to asymmetric radial density profiles due to the presence of an $\mathbf{E} \times \mathbf{B}$ drift [24]. When the magnetic field is reversed, the IV curves of the planar probes are also observed to reverse. This is strong evidence that $\mathbf{E} \times \mathbf{B}$ drifts are responsible for the transport of electrons across the filter, and hence for the presence of co-extracted electrons previously observed in the downstream region. The slightly different measured current magnitudes (and shapes of the IV curves), for the corresponding original and reversed magnetic field 
configurations are most likely due to the fact that the gas injection holes are located on only one side of the source, and so a small asymmetry is always present in the source operation. Similar results to those in Fig. 6 are also obtained for other gas flow rates between 0.5 - 8 SCCM.

Based on the above results, it is interesting to ask whether the presence of coextracted electrons change the results of the beam neutralization measurements in Section 3.3. To investigate this, we block about one-third of the extraction grids on the side of the original plasma source where electrons are present (i.e. the RHS for the original magnetic field configuration) with a thin metal sheet to minimise electron loss there. With this change, no visible downstream plasma can be seen anymore during both DC negative ion extraction, and alternate ion extraction. Figure 7 again shows the temporal variation of the target floating potential for applied frequencies of $20 \mathrm{kHz}$ and $250 \mathrm{kHz}$ respectively. Now at the low frequency, the floating potentials are much more symmetric for the positive and negative ion extraction, and the target charges up to close to the applied voltage. At the high frequency, the floating potential is significantly lower, and close to zero. These results indicate that beam neutralization can occur even in the absence of co-extracted electrons and downstream secondary plasma production.

\section{Conclusions}

In the results above we have experimentally demonstrated the alternate extraction and acceleration of positive and negative ions from an upstream plasma source. In addition, we have shown that the net extracted charge from the source can be controlled by varying the applied square waveform duty cycle, and that downstream beam neutralization can be achieved at sufficiently high extraction frequencies $(\sim 200 \mathrm{kHz})$. The measured ion beam energies were observed to remain relatively constant with frequency, while the extracted beam current densities were found to decrease with frequency until a local minimum. Floating potential measurements of a downstream target have provided evidence for the presence of electrons in the downstream region, which are co-extracted along with the negative ions. The presence of these electrons was confirmed in the upstream plasma source between the magnetic filter and the extraction grids, and they were found to be localized to one side of the source. This side depends on the orientation of the filter magnetic field, and corresponds to an $\mathbf{E} \times \mathbf{B}$ drift directed towards the grids, which results from an interaction of the sheath/pre-sheath electric field and the applied filter magnetic field. Thus these tests demonstrate that co-extracted electrons occur because of a non-closed $\mathbf{E} \times \mathbf{B}$ drift as predicted by PIC simulations [24]. By blocking a portion of the screen grid, electron co-extraction was observed to be strongly suppressed, and downstream neutralization of the positive and negative ion beams could still be achieved.

Future challenges now remain to optimize the present proof-of-concept device for specific industrial or propulsion applications. In particular, while the present experiments have validated the PEGASES thruster concept, the current system 
performance is very low, with a propellant utilization and thruster electrical efficiency of only a few percent [27]. This poor performance is due in large part to the use of relatively high gas flow rates, low applied discharge powers, and a large grid separation distance (which gives calculated space-charge limited current densities more than 3 times lower than those avalabile in the upstream plasma region), all chosen for experimental convenience.

\section{Acknowledgements}

The authors would like to thank S. Dudin at KhNU, Ukraine, for the design and construction of the single-phase invertor. This work received financial state aid managed by the Agence Nationale de la Recherche as part of the program "blanc" under the reference ANR-2011-BS09-40 (EPIC), the program "Investissements d'avenir" under the reference ANR-11- IDEX-0004-02 (Plas@Par), and by a Marie Curie International Incoming Fellowship within the 7th European Community Framework (NEPTUNE PIIF-GA-2012-326054). 


\section{References}

[1] Samukawa S and Mieno T 1996 Plasma Sources Sci. Technol. 5132

[2] Economou D 2007 Appl. Surf. Sci. 2536672

[3] Donnelly V M and Kornblit A 2013 J. Vac. Sci. Technol A 31050825

[4] Ahn T H, Nakamura K and Sugai H 1996 Plasma Sources Sci. Technol. 5139

[5] Shibayama T, Shindo H and Horiike Yasuhiro 1996 Plasma Sources Sci. Technol. 5254

[6] Kanakasabapathy S K, Overzet L J, Midha V and Economou D 2000 Appl. Phys. Lett. 7822

[7] Midha V and Economou D 2001 J. Appl. Phys. 901102

[8] Subramonium P and Kushner M J 2004 J. Vac. Sci. Technol. A 22534

[9] Oudini N, Raimbault J L, Chabert P, Meige A and Aanesland A 2013 Phys. Plasmas 20043501

[10] Agarwal A, Rauf S and Collins K 2012 J. Appl. Phys. 112033303

[11] Walton S G, Leonhardt D, Fernsler R F and Meger R A 2003 Appl. Phys. Lett. 83626

[12] Bacal M, Bruneteau J and Devynck P 1988 Rev. Sci. Instrum. 592152

[13] Aanesland A, Meige A and Chabert P 2009 J. Phys.: Conf. Ser. 162012009

[14] Aanesland A, Bredin J, Chabert P and Godyak V 2012 Appl. Phys. Lett. 100044102

[15] Bredin J, Chabert P and Aanesland A 2013 Appl. Phys. Lett. 102154107

[16] Rafalskyi D, Popelier L and Aanesland A 2014 J. Appl. Phys. 115053301

[17] Stuhlinger E 1964 Ion propulsion for space flight (McGraw-Hill, New York)

[18] Goebel D M and Katz I 2008 Fundamentals of Electric Propulsion: Ion and Hall Thrusters (Wiley, New Jersey)

[19] Gilleo M A and Kash S W 1960 ARS Preprint 1157

[20] Ruiz M, Urdampilleta I, Bombardelli C, Ahedo E, Merino M and Cichocki F 2014 The FPr LEOSWEEP Project: Improving Low Earth Orbit Security With Enhanced Electric Propulsion Space Propulsion Conference 2014, 19-22 May, Cologne, Germany

[21] Levko D, Garrigues L and Hagelaar G J M 2014 J. Phys. D: Appl. Phys. 47045205

[22] Benhenni M, de Urquijo J, Yousfi M, Hernandez-Avila J L, Merbahi N, Hinojosa G and Eichwald O 2005 Phys. Review E $\mathbf{7 1} 036405$

[23] Rafalskyi D, Bredin J and Aanesland A 2013 J. Appl. Phys. 114213303

[24] Kolev St, Hagelaar G J M, Fubiani G and Boeuf J P 2011 Plasma Sources Sci. Technol. 21025002

[25] Boeuf J P, Claustre J, Chaudhury B and Fubiani G 2012 Phys. Plasmas 19113510

[26] Lafleur T and Aanesland A 2014 Phys. Plasmas 21063510

[27] Aanesland A, Rafalskyi D, Lafleur T, Grondein P, Chabert P, Mazouffre S, Renaud D, Garrigues L, Hagelaar G J M and Levko D 2014 Development and test of the negative and positive ion thruster PEGASES 50th AIAA/ASME/SAE/ASEE Joint Propulsion Conference \& Exhibit, 28-30 July 2014, Cleveland, Ohio 


\section{Figure captions}

Fig. 1: Schematic of the PEGASES plasma source showing the insulating Pyrex source tube, magnetic filter, rf antenna, and the dual-grid extraction system (which is connected to a square-wave voltage generator). The additional inserted electrode is connected to the screen grid, while the magnetized retarding field energy analyzer (MRFEA) is mechanically attached to the accel grid.

Fig. 2: (a) Peak positive and negative ion beam energies, and (b) positive and negative ion beam current densities, as a function of the applied square-wave frequency. The applied voltage amplitude is $350 \mathrm{~V}$ (horizontal dashed line in (a)). The inset figure in (a) shows the IF-DF for a square-wave frequency of $200 \mathrm{kHz}$.

Fig. 3: Normalized IF-DF measured approximately $10 \mathrm{~cm}$ downstream of the plasma source for a square-wave frequency of $200 \mathrm{kHz}$ and an applied voltage amplitude of $350 \mathrm{~V}$.

Fig. 4: Positive and negative ion beam current densities as a function of the applied square-wave duty cycle. The vertical dashed line indicates a 50\% duty cycle. The applied voltage amplitude is $350 \mathrm{~V}$.

Fig. 5: Floating potential on the beam target, and screen grid potential, as a function of time for applied square-wave frequencies of (a) $20 \mathrm{kHz}$, and (b) $250 \mathrm{kHz}$. (c) Maximum positive floating potential as a function of the applied square-wave frequency. For (a)-(c) the applied voltage amplitude is $260 \mathrm{~V}$.

Fig. 6: Measured current as a function of the applied planar probe bias voltage for the original and reversed magnetic field configurations, for the (a) LHS probe, and the (b) RHS probe. The schematic in the lower right corner of (a)-(b) represents the screen grid (when viewed from inside the plasma source looking towards the downstream region) together with the location of the two planar probes. The black probe indicates the active probe.

Fig. 7: Floating potential on the beam target, and screen grid potential, as a function of time for applied square-wave frequencies of (a) $20 \mathrm{kHz}$, and (b) $250 \mathrm{kHz}$. For these measurements one third of the grid extraction area (beginning at the RHS of the grid) has been covered to reduce co-extracted electrons. The applied voltage amplitude is $260 \mathrm{~V}$. 
Alternate extraction and acceleration of positive and negative ions from a gridded plasma source13

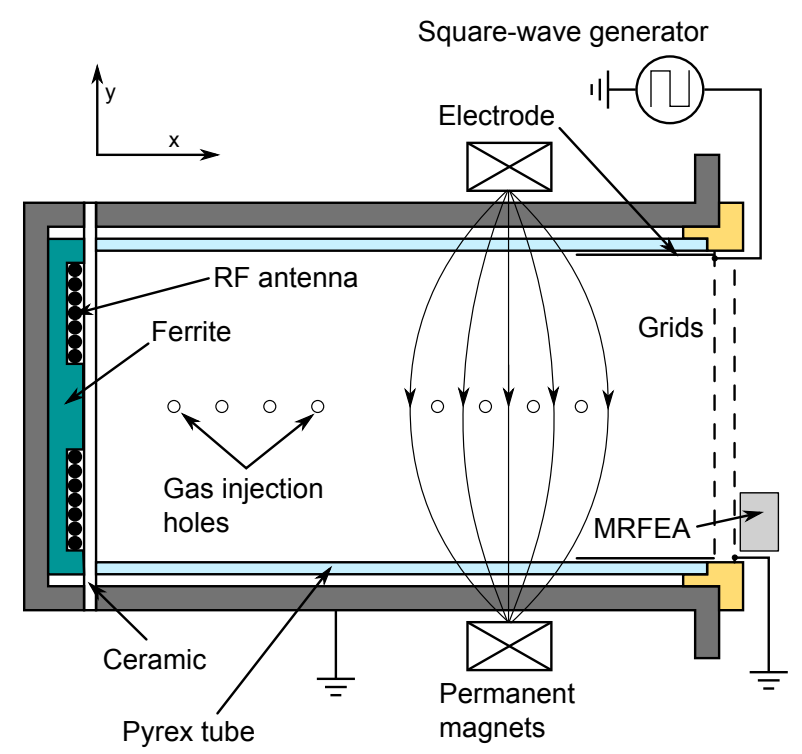

Figure 1.
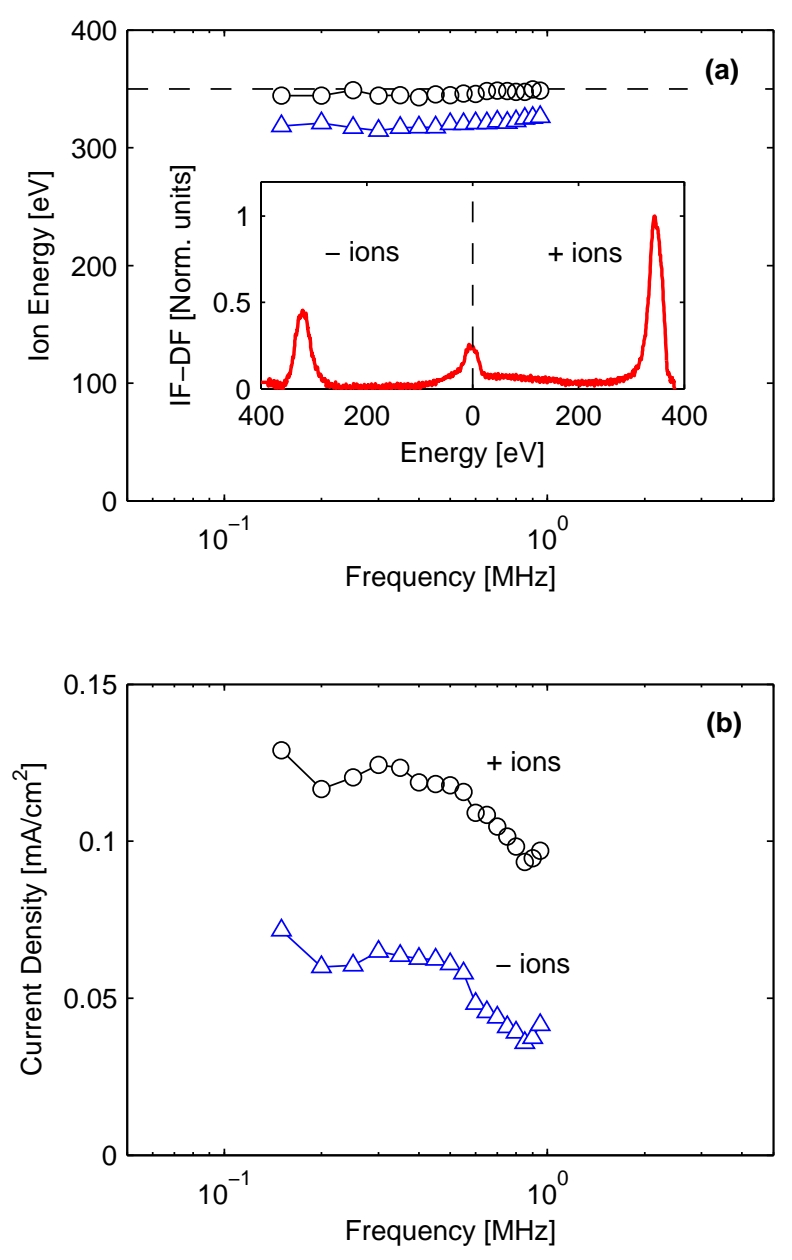

Figure 2. 
Alternate extraction and acceleration of positive and negative ions from a gridded plasma source 14

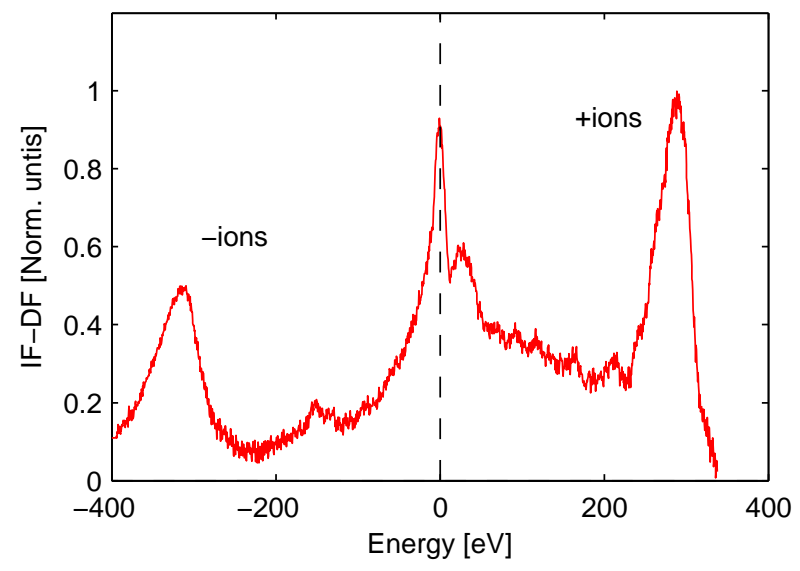

Figure 3.

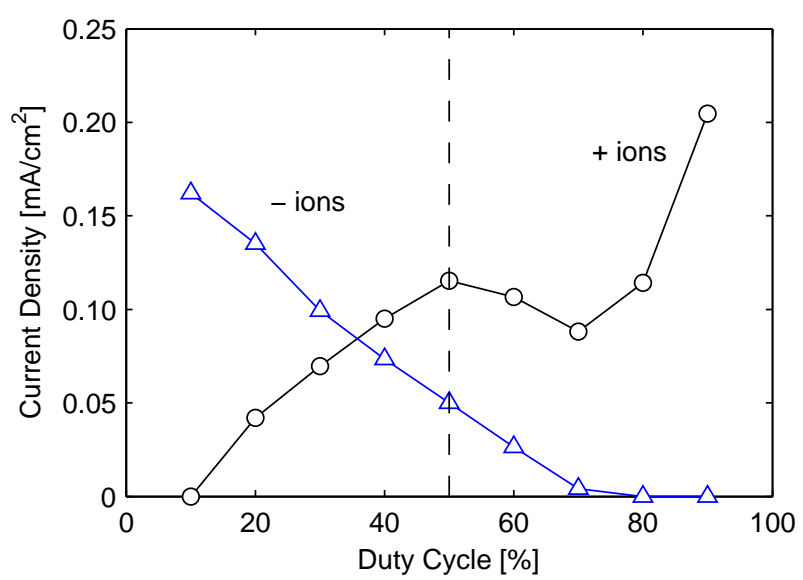

Figure 4. 

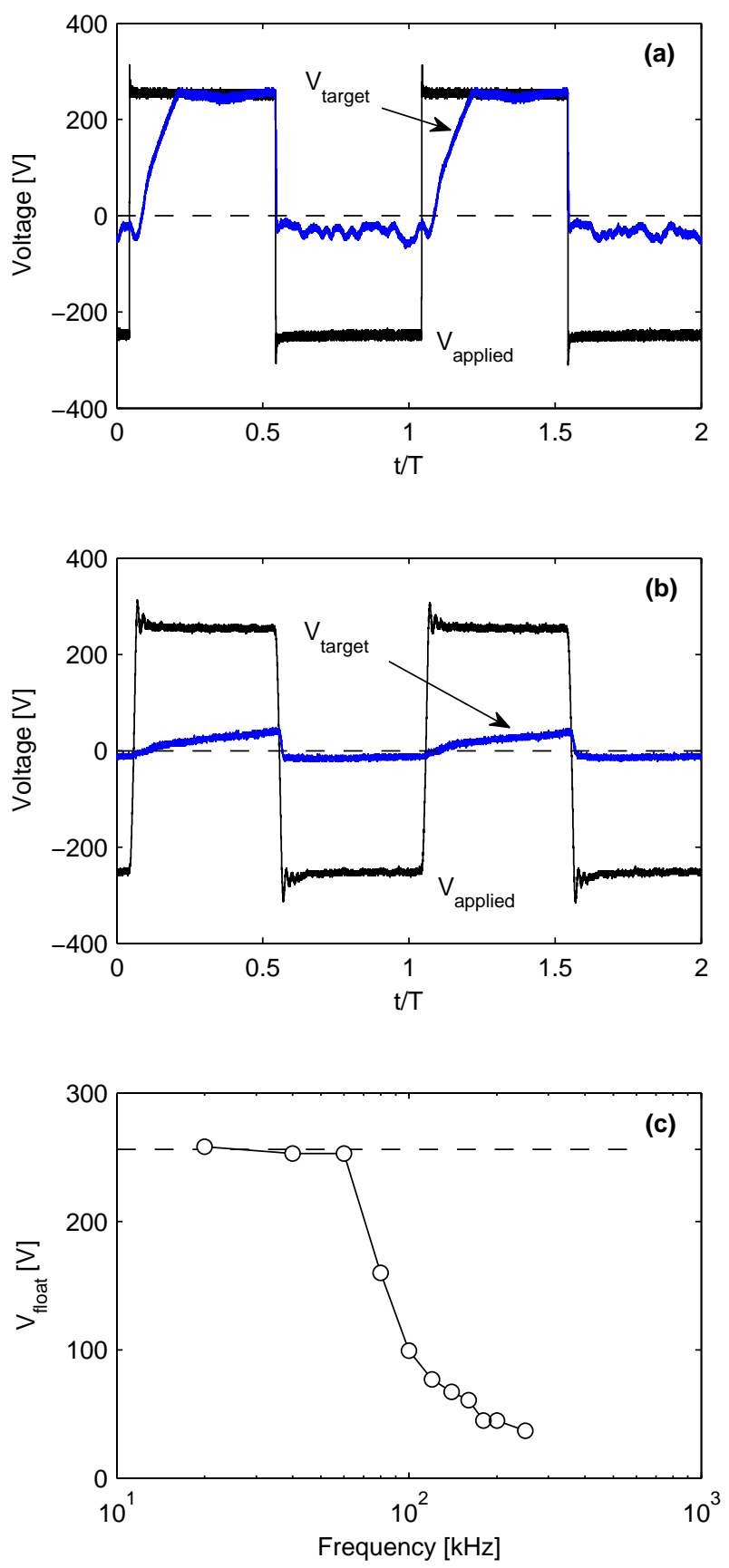

Figure 5. 

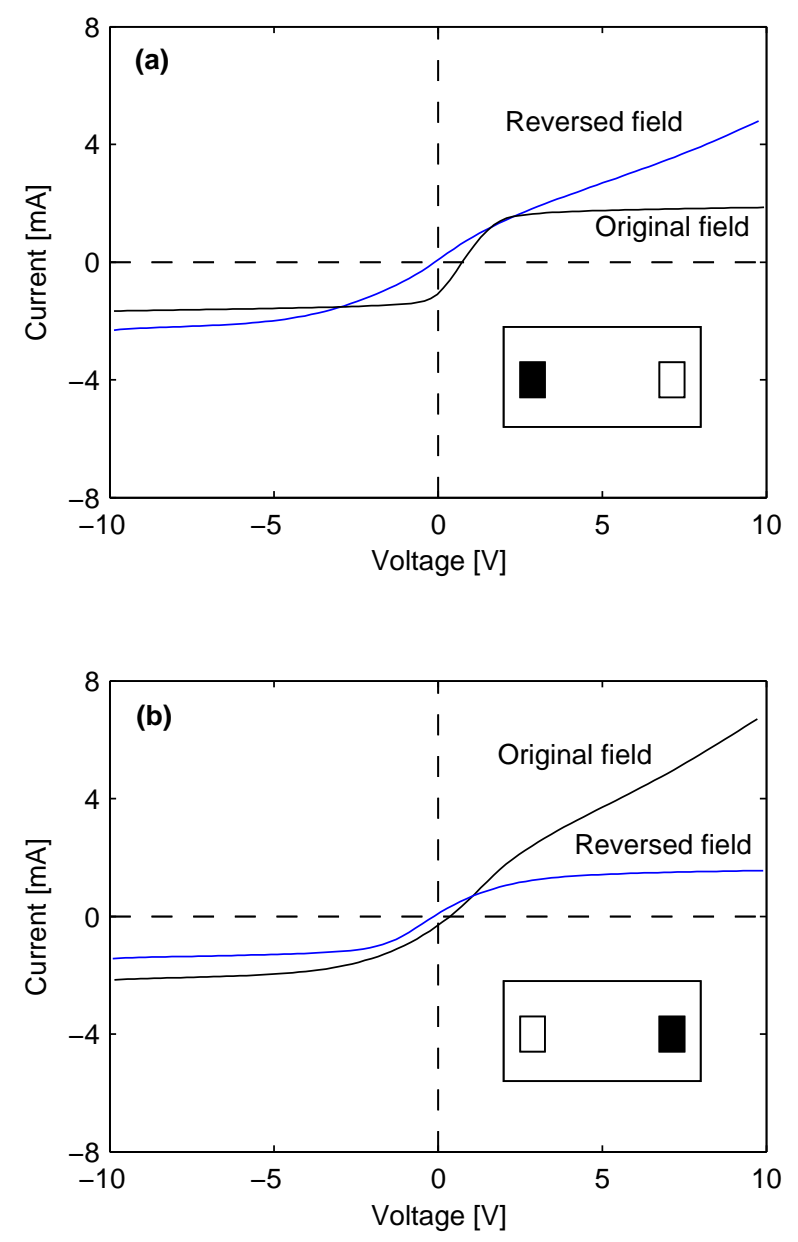

Figure 6. 

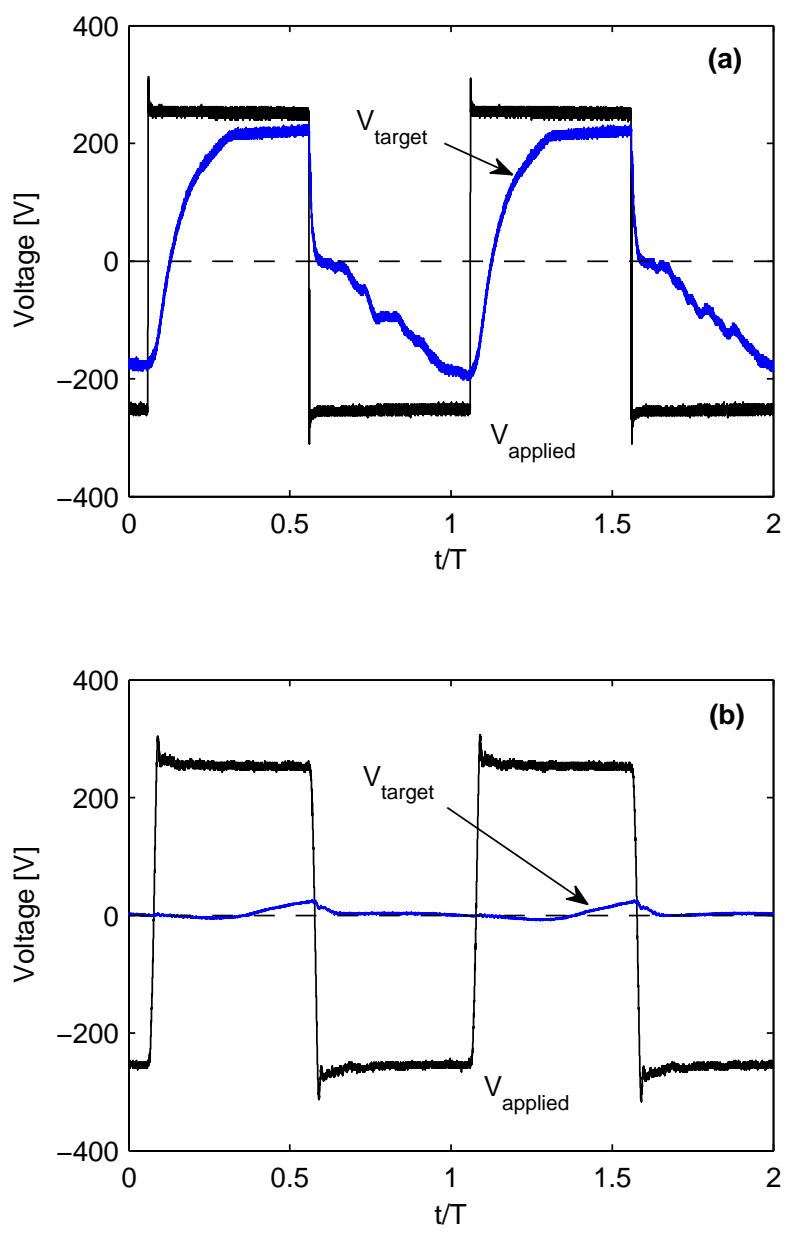

Figure 7. 